RECENSIONES 



\title{
GARCÍA FERRERO, A. (2012): IMAGEN Y PAISAJE, INSTRUMENTOS PARA LA PUESTA EN VALOR DEL TERRITORIO. LOS CASOS DE ARGANDA DEL REY Y EL BAJO TAJUÑA. ED. ACADÉMICA ESPAÑOLA. SAARBRÜCKEN (ALEMANIA). 200 PÁGS. ISBN: 978-3-659-00823-8
}

\author{
GARCÍA FERRERO, A. (2012): Image and landscape tools \\ for the enhancement of the territory. Cases of Arganda \\ del Rey and low Tajuña. Ed. Académica Española. Saarbrücken \\ (Alemania). 200 págs. \\ ISBN: 978-3-659-00823-87
}

\author{
Francisco José Morales Yago \\ Universidad Nacional de Educación a Distancia
}

\begin{abstract}
A través de un estudio local como serian los municipios madrileños de Arganda del Rey y el bajo Tajuña el autor de esta investigación plantea un análisis general del territorio centrado esencialmente en tres objetivos básicos. El primero de ellos sería la identificación del espacio vivido, señalando el hábitat y su evolución poblacional a lo largo del siglo XX y comienzos del actual siglo XXI. El segundo aborda de forma más profunda el comportamiento poblacional desde parámetros básicos como serían la evolución de la pirámide poblacional o diversos índices demográficos como son los indices de envejecimiento, natalidad o mortalidad. Finalmente el tercer y último objetivo de este trabajo estará encaminado a identificar los elementos que han protagonizado la economía y el sistema de transporte utilizado en el pasado y el vigente en la actualidad con un especial hincapié en la evolución del mismo.
\end{abstract}

En cuanto a su organización formal esta publicación se presenta en cinco capitulos los cuales al unirlos en su conjunto ofrecen un panorama muy completo de estudio y tienen como eje principal la visión del paisaje y su evolución patrimonial y cultural lo que ha repercutido en unas aceleradas y significativas transformaciones que deberian ayudar a la reflexión y puesta en valor de un espacio heredado con importantes potencialidades para sus habitantes $y$ los posibles visitantes que acudan a contemplarlo o deseen conocerlo de una forma científica.

En el primer capitulo titulado: "El medio y las gentes", se presenta este espacio en su faceta física y poblacional, destacando sin caer en determinismos como el medio físico ejerce una gran influencia en las personas y al mismo tiempo como estas evolucionan de forma progresiva o regresiva según la situación económica o la influencia de otros factores determinantes como la eficacia de la asistencia sanitaria, salubridad o el traslado hacia otros espacios. Llama la atención el contraste entre los distintos municipios que componen este espacio geográfico en cuanto a su evolución poblacional, por ejemplo el municipio de Arganda del Rey ha tenido según su autor un crecimiento "explosivo", mientras que los municipios de Morata de Tajuña, Perales y Tielmes lo han tenido más moderado, por el contrario otros como Carabaña, Orusco y Ambite han retrocedido en cuanto a su número de efectivos. La cercanía a la capital madrileña y la existencia o no de comunicaciones adecuadas han sido los factores 

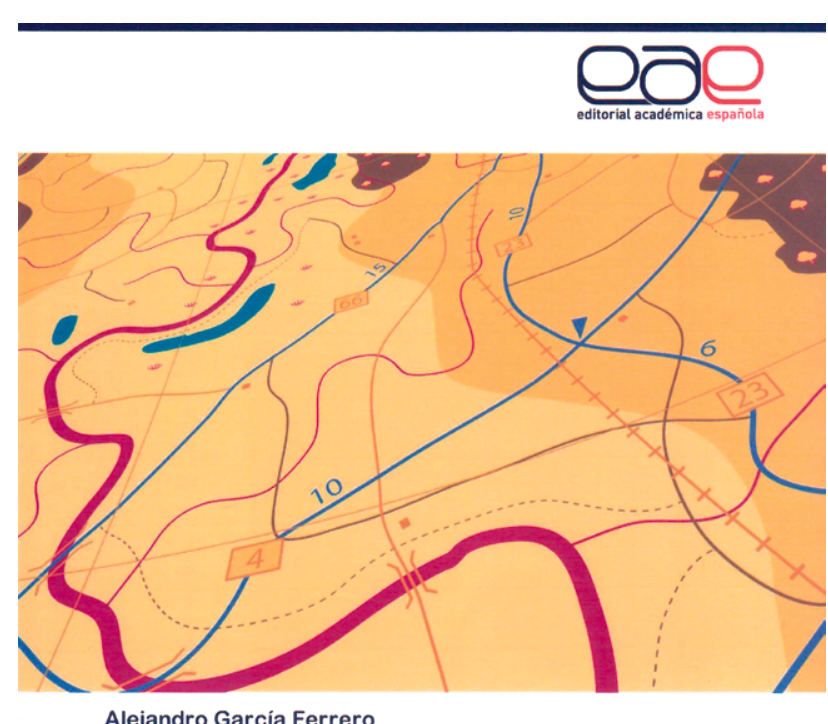

Alejandro García Ferrero

\section{Imagen y paisaje, instrumentos para la puesta en valor del territorio}

\author{
Los casos de Arganda del Rey y el bajo \\ Tajuña
}

determinantes en este proceso poblacional experimentado en los municipios estudiados.

El epígrafe del capítulo números dos señala "El espacio agrario" como el soporte esencial en la actividad económica de sus habitantes hasta épocas muy recientes, de hecho hasta la década de los 60 del pasado siglo XX alrededor del $80 \%$ de la población activa trabajaba en el medio rural, esencialmente en tareas como el cultivo de la vid, aceite de oliva y obtención de azúcar. Dada la proximidad al núcleo de la capital madrileña siempre ha existido una amplia demanda de estos productos y la seguridad por tanto de que las cosechas han tenido una salida relativamente fácil ha estado asegurada. También una ganadería basada en el abastecimiento de carnes como el bovino, porcino y aves ha tenido en Madrid el principal referente para las ventas y mantenimiento de su mercado. No obstante en las últimas décadas la superficie agraria se ha visto reducida por la aparición de la actividad industrial y el uso del suelo para nuevos espacios residencial, en algunos casos como viviendas principales y en otros como segundas residencias, la mayoría de ellos de personas provenientes de Madrid que dada la cercanía han buscado un lugar de descanso en régimen de fin de semana o para el periodo estival.

El capítulo tercero se ocupa de un sector más reciente en el tiempo que ha ido ganando importancia y peso respecto al espacio agrario y que en la actualidad se ha convertido en uno de los referentes principales en la creación de trabajo y bienestar como es la industria y el comercio. La industria derivada del azúcar así como las manufacturas del comercio "al detalle" han sido elementos clave en la economía local, señala el autor ejemplos muy concretos como la azucarera de "La Poveda" u otras instalaciones industriales tan diversas como fábricas de mantas, harinas, papel, algodón o centrales eléctricas, sin olvidar la explotación del famoso manantial de aguas minerales de Carabaña.

La red de transporte ocupa los contenidos del capítulo cuarto. De forma amena y suficientemente amplia a lo largo de este capítulo se relatan los esfuerzos realizados por crear una red de carreteras adecuada que vertebrara el espacio así como la trascendencia del transporte ferroviario que finalmente se ha visto sobrepasado por el transporte a través de la carretera, mucho más rápido y eficaz que este, de hecho el avance de los transporte por este medio fue la principal causa del desmantelamiento de la red de ferrocarriles la cual quedó obsoleta y definitivamente reducida al transporte solamente de personas, hecho que se generó la ampliación de la línea de metro número nueve hasta Arganda del Rey, con un buen servicio a espacios como las distintas estaciones del municipio de Rivas-Vaciamadrid y La Poveda.

El capítulo quinto, el último de forma muy breve presenta un importante apartado dedicado a los "Nuevos usos del paisaje", a través del mismo se identifican los elementos que han repercutido en el cambio de un paisaje tradicional hacia un nuevo modelo urbano, en donde se combina lo establecido como la tipología de casa manchega o alcarreña con nuevas construcciones funcionales y adaptadas a los nuevos tiempos que en muchos casos se produjo de forma poco planificada, casi espontanea; que ha generado la creación de espacios impersonales y de gran impacto en el paisaje que lejos de embellecerlo lo han degradado como por ejemplo la urbanización de Valdeperales en el municipio de Perales de Tajuña.

Finalmente en las conclusiones, el autor aporta un conjunto de ideas en donde de forma sintética concreta los principales resultados de la investigación efectuada, señalando que la historia reciente de este espacio es un fiel reflejo de las transformaciones experimentadas por la región madrileña como consecuencia del crecimiento acaecido en Madrid y su área metropolitana lo que ha tenido repercusiones en aspectos diversos y al mismo tiempo complementarios como serian las dinámicas de la población, el transporte o la propia actividad económica de sus habitantes lo que no cabe dudas incide directamente en la transformación del paisaje tanto física como culturalmente.

Muy destacable en el trabajo de García Ferrero es la abundante documentación gráfica aportada en el trabajo presentado: trece mapas, siete planos, veintidós tablas, trece gráficos y sesenta ilustraciones hacen de esta investigación una publicación amena, donde la imagen complementa per- 
fectamente a las palabras y amplía notablemente el conocimiento del espacio que el autor pretende mostrar, se hace un gran elenco y recuperación de fotografías del presente y pasado, una cartografía abundante que rescata mapas del Instituto Geográfico Nacional hasta las fotografías más recientes extraídas desde el programa Google Earth así como la información ofrecida por la Comunidad de Madrid y ayuntamientos objeto de estudio. También es importante señalar la amplia bibliografía aportada, la cual se encuentra actualizada y a disposición para otros posibles estudios que desde la escala local pueden pasar a la global, ya que la metodología empleada es válida para este tipo de investigaciones, esencialmente el enlace a distintas páginas web.

Esta publicación muestra la experiencia acumulada por el autor sobre el conocimiento de este espacio madri- leño cuyas singularidades son destacadas de forma ejemplar y con la especial sensibilidad que aporta la ciencia geográfica en el análisis del espacio geográfico así como la reflexión que ello conlleva para la planificación y ordenación futura del territorio, aportando potencialidades y posibilidades de llevar a cabo una gestión sostenible que no cabe dudas redundará en la puesta en valor de dicho territorio.

No queda más que dar la enhorabuena al autor e invitarle a que continúe periódicamente publicando sus investigaciones que tanto enriquecen al quehacer de la ciencia geográfica.

Francisco José Morales Yago Universidad Nacional de Educación a Distancia 
